\title{
Yeast need not be used only in beer production. I. The carotenoid astaxanthin produced by yeast.
}

\author{
Tomáš Řezanka ${ }^{1 *}$, Andrea Palyzová ${ }^{1}$ \\ 1 Institute of Microbiology, Academy of Sciences of the Czech \\ Republic, Vídeňská 1083, 14220 Prague, Czech Republic
}

*corresponding e-mail: rezanka@biomed.cas.cz

\begin{abstract}
Carotenoids are produced by photosynthesizing organisms, i.e. plants and algae, as well as some bacteria, protists and fungi. Their role lies mainly in the function of complementary photosynthetic pigments and antioxidants. $\mathrm{Ca}-$ rotenoids are distinguished according to their structure into carotenoids of the hydrocarbon type, e.g. $\alpha-, \beta-$ and $\gamma$-carotene and oxygen derivatives - xanthophylls, among which astaxanthin, which excels in its antioxidant and nutraceutical properties, is the most important. It is used as a dietary supplement both in human nutrition and in the aquaculture and poultry industry, as a nutritional supplement in salmonid and hen farming. Many works have focused on the search for natural sources of astaxanthin as an alternative to synthetic astaxanthin. In nature, very few astaxanthin producing organisms are known in non-minor amounts, with green alga Haematococcus pluvialis and the yeast Xanthophyllomyces dendrorhous (anamorph Phaffia rhodozyma) being the most promising microorganisms for the biotechnological production of this xanthophyll. In this review we focus on the biosynthesis and carotenogenesis including the regulation mechanisms, generation of hyperproducing mutants by random mutagenesis and, last but not least, also metabolic engineering (genetic techniques). Attention is also paid to the optimization of fermentation conditions.
\end{abstract}

Keywords: yeast, Xanthophyllomyces dendrorhous, Phaffia rhodozyma, astaxanthin

\section{Introduction}

Astaxanthin belongs to carotenoids, i.e. compounds derived from tetraterpenes having 40 carbon atoms. Structurally and functionally, carotenoids can be classified as hydrocarbons (carotenes) and oxygen derivatives (xanthophyls), i.e. compounds containing hydroxyl and a ketone as a functional group. Astaxanthin is red because it contains a chain of conjugated double bonds. This chain of conjugated double bonds is also responsible for the antioxidant function of astaxanthin (as well as other carotenoids).

Astaxanthin is the third economically most important carotenoid after $\beta$-carotene and lutein. The astaxanthin market reached $29 \%$ of total carotenoid sales, with an estimated global market size of \$225 million (Mata-Gomez et al., 2014).
Astaxanthin is found above all in primary sources in some algae and yeasts and then in secondary sources marine invertebrates (small crustacean plankton (krill), shrimp, crayfish, etc.), salmon, trout and waterfowl feathers. It is the cause of the red color of salmon and the red color of cooked crustaceans. Astaxanthin, unlike some carotenes, is not a precursor to vitamin A. Like other carotenoids, astaxanthin is poorly absorbed orally and no toxic syndrome is known. It has high antioxidant effects due to its conjugated chain and its solubility in polar solvents (e.g. water) is higher due to four functional groups ( $2 x$ ketone and $2 x$ hydroxyl) than that of e.g. $\beta$-carotene.

Astaxanthin is used as a food supplement both in humans and animals and in aquaculture. Commercial astaxanthin comes from both natural and synthetic sources. 
Astaxanthin is approved by both the US Food and Drug Administration (FDA) and the European Commission (E161j). Among the uses of astaxanthin is, e.g. its use for improving pigmentation of yolk and poultry color (Elwan et al., 2019).

The sources of astaxanthin are either sea krill (see above) or cultivated algae (mainly Haematococcus pluvialis) or yeasts, e.g. X. dendrorhous, formerly P. rhodozyma.

The $X$. dendrorhous taxonomy was determined in 2015 and 2016 for two strains based on the entire genome sequence, ranging in size from 19.1 to $19.5 \mathrm{Mb}$ (GenBank LN483142.1 and BCJZ01000001.1).

Currently, the main natural source of astaxanthin is the microalga Haematococcus pluvialis. Commercially, more than $40 \mathrm{~g}$ of astaxanthin can be obtained from one $\mathrm{kg}$ of dry biomass. Cultivation takes place in two phases; in the first phase there is an increase in biomass, in the second phase the alga is cultivated under nutrient starvation and strongly irradiated by visible light. In the case of cultivation in nature, the cultivation is limited to the tropical belt and places with sufficiently long sunshine. In addition, the algae produce not only astaxanthin but also mainly its esters and diesters, which must be chemically hydrolyzed before being administered as a dietary supplement. Yeasts, which do not have these drawbacks, can be grown in the dark. In addition, they produce only free astaxanthin and yeast fermentation has been industrially mastered in hundreds of thousands of liters (e.g. beer production). Astaxanthin has two centers of chirality, is symmetric and therefore occurs in 3 isomers (3R, 3'R), (3R, 3'S), and (3S, 3'S). Synthetic astaxanthin contains a mixture of all three isomers in a ratio of 25:50:25. Natural astaxanthin also contains all three isomers, but not in a 25:50:25 ratio. Astaxanthin produced by algae, e.g. freshwater Haematococcus pluvialis, produces more than $90 \%$ of the isomer $3 \mathrm{~S}$, 3'S, while the yeast Xanthophyllomyces dendrorhous produces more than $90 \%$ of the $3 \mathrm{R}, 3$ ' $\mathrm{R}$ isomer, see Figure 1. Therefore, in contrast to salmon caught in the wild, it is very easy to prove that farmed salmon fed with synthetic astaxanthin has been artificially fed with it. The price of synthetic astaxanthin is several times lower than that of the compound isolated from algae and yeast (the price of synthetic astaxanthin ranges from about $\$ 5,000$ to $\$ 6,000$ per $\mathrm{kg}$, according to other sources it can be up to $\$ 7,000 / \mathrm{kg}$ from (http://www.astaxanthin.company).

\section{Organic synthesis}

Total astaxanthin synthesis is the cheapest method to obtain it on an industrial scale. It has been very successful - see for example, the book describing organic synthesis, including hundreds of intermediates (Britton et al., 1996). The disadvantage of this synthesis, as mentioned above, is the mixture of three isomers. Though the synthesis of both high purity 3R, 3'R and/or 3S, 3'S enantiomers has been mastered, the cost of the resulting product is many times higher due to the use of special chiral catalysts based on the central ruthenium atom complexed with an optically active amine (Lockwood et al., 2007). Another possibility of obtaining an optically active isomer is the separation of the three isomers on

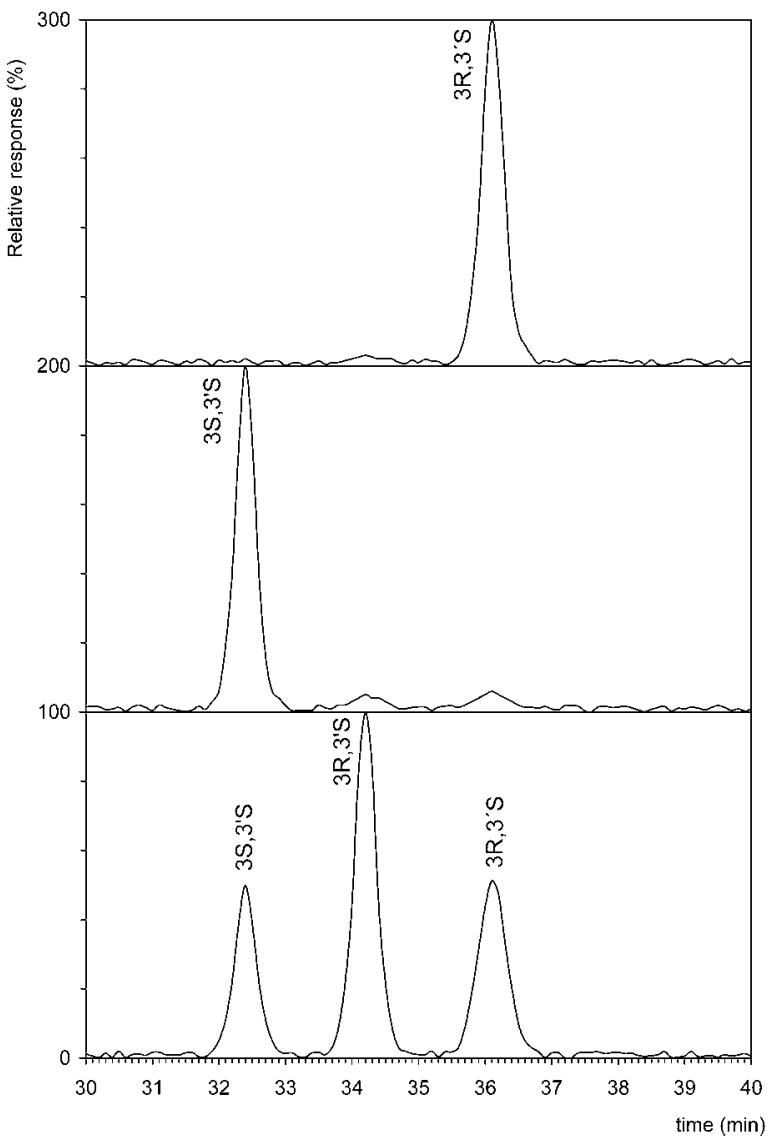

Figure 1 HPLC comparison of astaxanthin isomers found in alga, yeast and synthetic astaxanthin. Column CHIRALCEL $\AA$ OD-RH, $150 \times 4,6 \mathrm{~mm}, 5 \mu \mathrm{m}$ was used with mobile phase acetonitrile/phosphoric acid ( $3.5 \mathrm{mM}$ ) at a flow rate of $0.5 \mathrm{ml} / \mathrm{min}$ under isocratic conditions and a temperature of $25^{\circ} \mathrm{C}$. Identification of astaxanthin peaks was achieved by using the wavelength of $470 \mathrm{~nm}$ (Rezanka et al., 2013). 
a preparative chiral column, but for economic reasons (the cost of a column with such a special chiral phase), this procedure is currently not feasible.

\section{Biosynthesis}

Carotenoid biosynthesis probably originates from ancient anoxygenic and photosynthetic microorganisms, such as cyanobacteria, that are up to 3.5 billion years old. The change of the Earth's atmosphere from anaerobic to aerobic is the main factor responsible for the biosynthesis of structurally diverse carotenoids, using oxygen-dependent enzymes (Liang et al., 2006).

The biosynthetic pathway of carotenoids in eukaryotic cells includes the following reactions (Mata-Gomez et al., 2014). The synthesis begins with the condensation of acetyl-CoA to 3-hydroxy-3-methylglutaryl-CoA (HMG-CoA), which is catalyzed by HMG-CoA synthase. Then, HMG-CoA produces mevalonic acid (MVA), the first ancestor of the terpenoid biosynthetic pathway. The following steps include two sequential phosphorylation reactions performed by mevalonate kinase and phosphomevalonate kinase, and the final step is phosphomevalonate decarboxylase catalyzed decarboxylation to produce isopentenyl pyrophosphate (IPP, or isopentenyl diphosphate, IDP) (Mata-Gomez et al., 2014; Werner et al., 2016). IPP is isomerized to dimethylallylpyrophosphate (DMAPP). Reaction of three IPP molecules with DMAPP, catalyzed by prenyltransferases, yields geranylgeranyl pyrophosphate (GGPP). The condensation of two GGPP molecules produces phytoene, the first C40 carotene, which is subsequently desaturated to form lycopene (Mata-Gomez et al., 2014). Lycopene undergoes many modifying and cyclic reactions leading to the biosynthesis of compounds such as $\beta$-carotene, $\gamma$-carotene, torulene, torularhodin and astaxanthin (Mata-Gomez et al., 2014). This general overview varies depending on the microorganisms. Only astaxanthin biosynthesis in X. dendrorhous will be discussed below.

\subsection{Microbial production of astaxanthin in X. dendrorhous}

It is currently believed that the main function of carotenoids in $X$. dendrorhous is to protect yeast from the damage caused by oxidative stress. This hypothesis is supported by the fact that strains that do not produce astaxanthin are more sensitive and grow less in the presence of reactive oxygen species (ROS) (Schroeder and Johnson, 1993). Singlet oxygen and peroxyl radicals have been reported to increase total carotenoid content (Schroeder and Johnson, 1993; Schroeder and Johnson, 1995). Furthermore, $X$. dendrorhous has significantly lower catalase activity than, for example, Saccharomyces cerevisiae, so the low enzyme activity can be compensated by carotenoid biosynthesis (Schroeder and Johnson, 1993), since superoxide dismutase is also not present in the cytosol of $X$. dendrorhous, and carotenoids could again compensate for its deficiency (Schroeder and Johnson, 1993). Meanwhile, $X$. dendrorhous is the only known yeast producing astaxanthin de novo (Ambati et al., 2014).

Lycopene is converted to $\beta$-carotene by the bifunctional enzyme phytoene- $\beta$-carotene synthase (PBS) having lycopene cyclase activity. Finally, $\beta$-carotene is oxidized by incorporating the hydroxyl group at the 3-position and the keto group at the 4-position on both $\beta$-ionic rings to form astaxanthin as the final product. Unlike other organisms that produce astaxanthin, in $X$. dendrorhous these last oxidation steps from $\beta$-carotene to astaxanthin are catalyzed by a single enzyme called astaxanthin synthase (CrtS, encoded by crtS gene), a cytochrome P450 monooxygenase (Ojima et al., 2006). Astaxanthin synthase requires a redox partner, the cytochrome P450 reductase encoded by the $X$. dendrorhous crtR gene (Alcaíno et al., 2008; Alcaíno et al., 2012; Gutiérrez et al., 2015), which provides the necessary electrons for enzymatic catalysis. The outcome of the biosynthesis can be summarized as the production of xanthophylls with astaxanthin in between 80 and $90 \%$ of total carotenoids (Schmidt et al., 2011).

Biosynthesis of astaxanthin in H. pluvialis proceeds similarly, though different biosynthetic steps, including esterification of one or both hydroxyl groups, have been described (Lorenz and Cysewski, 2000).

In addition to these two major microorganisms producing carotenoids, Brevibacterium linens, Agrobacterium aurantiacum and marine bacterium Paracoccus haeundaensis are also listed for the production of astaxanthin, but are not considered commercially important sources (Vachali et al., 2012).

$X$. dendrorhous has two evolutionary special metabolic features. One is the synthesis of astaxanthin, which is considered unique among fungi. The other is the fermentation of sugar to alcohol under oxidative conditions (Reynders et al., 1997). Astaxanthin serves as an antioxidant, quenching reactive oxygen species to protect $X$. dendrorhous from damage by oxidative stress (William and Schroeder, 1993; William and Schroeder, 1995). Its biosynthesis is via the mevalonate pathway to the formation of $\beta$-carotene with enzymes similar to other carotenogenic fungi (Sandmann, 2002). However, all steps of 3-hydroxylation and 4-ketolation at both terminal $\beta$-ionone rings leading to the formation of astaxanthin are carried out by a very unique P450 monooxygenase. This protein, Asy, belongs to the $3 \mathrm{~A}$ subfamily (Ojima et al., 2006); electrons are provided by a specific cytochrome 
P450 reductase (Alcaíno et al., 2008). Although the astaxanthin concentration in wild-type strains of $X$. dendrorhous is too low for commercialization, attempts have been made to increase the astaxanthin yield, developing $X$. dendrorhous as a production system for this carotenoid. The most promising yields were obtained by a combination of classical random mutagenesis followed by systematic engineering of the whole biosynthesis pathway (Gassel et al., 2014).

\section{Biotechnological production of astaxanthin}

First and foremost, for economic reasons, it is important to both optimize fermentation parameters and improve the ability of wild-type yeast strains to produce industrially used astaxanthin. Therefore, low-cost culturing media such as alfalfa residual juice, cane molasses, sugar cane juice, corn wet-milling co-products, grape juice, hydrolyzed peat, raw coconut milk or vegetable oils were used. The nutrient-rich culture medium and the presence of astaxanthin precursors have generally been shown to be favorable to increased astaxanthin production. It has also been reported that carotenogenesis in many organisms is regulated by light. An et al. (1996) investigated the effect of oxygen radicals and aeration on the carotenogen- esis and growth of $P$. rhodozyma and found increased astaxanthin production. Kim et al. (2003) report that both biomass and astaxanthin production are stimulated by the addition of ethanol $\left(10 \mathrm{~g} \mathrm{~L}^{-1}\right)$ and acetic acid $\left(5 \mathrm{~g} \mathrm{~L}^{-1}\right)$ to a culture of $P$. rhodozyma, resulting in a respective yield of $45.62 \mathrm{mg} \mathrm{L}^{-1}$ and $43.87 \mathrm{mg} \mathrm{L}^{-1}$ of carotenoids. Similarly, $\mathrm{Gu}$ et al. (1997) found increased carotenoid production (from $1.65 \mathrm{mg} \mathrm{g}^{-1}$ carotenoids to $2.65 \mathrm{mg} \mathrm{g}^{-1}$ carotenoids) by adding $0.2 \%$ ethanol to $X$. dendrorhous yeast cultures.

Various approaches have been taken to enhance the content of astaxanthin in Phaffia, such as genetic manipulation (An et al., 1989; Fang and Chiou, 1996; Meyer and du Preez, 1994) and protoplast fusion (Chun et al., 1992). Another possibility is to use genetically modified yeast. Using wild strains, conventional mutagenic agents, including, e.g. the mutagenic effects of $N$-methyl- $N$ '-nitro- $N$-nitrosoguanidine, hydrogen peroxide, ethidium bromide or UV irradiation and/or $\gamma$-rays have been successfully used to isolate various strains producing astaxanthin.

The greatest potential approach is the cloning and overexpression of the carotenoid biosynthetic genes of the Phaffia/Xanthophyllomyces strains, see Table 1.

Table 1 gives examples of successful mutagenesis, i.e. the use of both cloning and expression of carotenoid biosynthetic genes in Phaffia/xanthophyllomyces strains. Chemical mutants with significantly higher astaxanthin

Table 1 Summary of astaxanthin production by natural producers and engineered non-carotenogenic organisms. Single black arrow ( $\downarrow$ ) represents downregulation.

\begin{tabular}{|c|c|c|c|}
\hline Organism & Genotype & $\begin{array}{l}\text { Astaxanthin production } \\
\text { and type of cultivation }\end{array}$ & Reference \\
\hline X. dendrorhous & crtYB and asy (native genes) & 9.7 mg/g DCW (bioreactor) & Gassel et al., 2013 \\
\hline X. dendrorhous & crtYB, asy, crtE and trHMG (native genes) & 9 mg/g DCW (shake-flasks) & Gassel et al., 2014 \\
\hline H. pluvialis & site-directed mutagenesis of PDS (native gene) & 11.4 mg/g DCW (shake-flasks) & $\begin{array}{l}\text { Steinbrenner and Sandmann, } \\
2006\end{array}$ \\
\hline E. coli & $\begin{array}{l}\text { crtE, crtY, crtl, crtB, crtZ (from P. ananatis); trBKT } \\
\text { (from C. reinhardtii); ispD and ispF (native genes) }\end{array}$ & $\begin{array}{l}432 \mathrm{mg} / \mathrm{L}, 7 \mathrm{mg} / \mathrm{g} \mathrm{DCW} \\
\text { (bioreactor) }\end{array}$ & Park et al., 2018 \\
\hline E. coli & $\begin{array}{l}\text { Module 1: atoB (native), hmgS (S. cerevisiae), and } \\
\text { thmgR (S. cerevisiae); } \\
\text { Module 2: mevk (S. cerevisiae), pmk (S. cerevisiae), } \\
\text { pmd (S. cerevisiae), and idi (native); } \\
\text { Module 3: crtEBI (amplified from pAC-LYC } \\
\text { plasmid) and ispA (native); crtY (P. ananatis), crtZ } \\
\text { (from P. ananatis), crtW (Brevundimonas sp.) }\end{array}$ & $\begin{array}{l}320 \mathrm{mg} / \mathrm{L}, 2 \mathrm{mg} / \mathrm{g} \mathrm{DCW} \\
\text { (simultaneous fermentation } \\
\text { and extraction) }\end{array}$ & Zhang et al., 2018 \\
\hline S. cerevisiae & $\begin{array}{l}\text { crtW (from Brevundimonas vesicularis),crtZ (from } \\
\text { Agrobacterium aurantiacum), and mutagenesis of } \\
\text { CSS1, YBR012W-B and DAN4 }\end{array}$ & $\begin{array}{c}217.9 \mathrm{mg} / \mathrm{L}, 13.8 \mathrm{mg} / \mathrm{g} \mathrm{DCW} \\
\text { (bioreactor) }\end{array}$ & Jin et al., 2018 \\
\hline S. cerevisiae & $\begin{array}{l}\text { crtE, crtl, crtYB (from } X . \text { dendrorhous); trHMG1 } \\
\text { (native gene); BKT and crtZ (from H. pluvialis) }\end{array}$ & $\begin{array}{l}47 \mathrm{mg} / \mathrm{L}, 8 \mathrm{mg} / \mathrm{g} \mathrm{DCW} \\
\text { (shake-flasks) }\end{array}$ & Zhou et al., 2017 \\
\hline S. cerevisiae & $B K T$ and $c r t Z$ (from $H$. pluvialis) & $\begin{array}{l}4.7 \mathrm{mg} / \mathrm{g} \mathrm{DCW} \\
\text { (shake-flasks) }\end{array}$ & Zhou et al., 2015 \\
\hline Y. lipolytica & $\begin{array}{c}\text { crtYB, crtl, crtE (from } X \text {. dendrorhous); HMG1 } \\
\text { (native gene); } \downarrow S Q S 1 ; \text { crtW (from Paracoccus sp.) } \\
\text { and crtZ (from P. ananatis) }\end{array}$ & $\begin{array}{l}54.6 \mathrm{mg} / \mathrm{L}, 3.5 \mathrm{mg} / \mathrm{g} \mathrm{DCW} \\
\text { (microtiter plates) }\end{array}$ & Kildegaard et al., 2017 \\
\hline Y. lipolytica & $\begin{array}{c}\text { GGPPs7 (from Synechococcus sp.), HpBKT, } \\
\text { HpcrtZ (from H. pluvialis) }\end{array}$ & $\begin{array}{l}285 \mathrm{mg} / \mathrm{L}, 6 \mathrm{mg} / \mathrm{g} \mathrm{DCW} \\
\text { (bioreactor) }\end{array}$ & Tramontin et al., 2019 \\
\hline
\end{tabular}


production were produced. To further improve astaxanthin production, it is an advantage that in $X$. dendrorhous, carotenoid biosynthesis can be genetically manipulated. All necessary pathway genes were cloned. They include those encoding a 3-hydroxymethyl-3-glutaryl coenzyme A reductase, geranylgeranyl pyrophosphate synthase, phytoene synthase/lycopene cyclase, and astaxanthin synthase. Combination of classical mutagenesis with genetic engineering has improved the flow of metabolites into the carotenoid pathway and the efficient conversion of intermediates to the desired end product astaxanthin. Starting with two mutants with a 15-fold higher astaxanthin content, transformants were obtained with a further 6-fold increase in the last step of the biosynthetic pathway. Thus, a maximum astaxanthin content of almost $9 \mathrm{mg}$ per $\mathrm{g}$ dry weight (Gassel et al., 2014) was achieved in shake cultures.

A very sophisticated approach to increase astaxanthin production was to use multivariate modular methods to optimize astaxanthin production, which allows reducing regulatory complexity by grouping multiple genes into modules. Unfortunately, there was a shortcoming of this method in that it works well for balancing the inter-modules but not the intra-modules. Therefore, to further increase astaxanthin production, a multidimensional heuristic process (MHP) has been developed. At the same time, MHP balances different modules by changing promoter potency and coordinating activities within the module using ribosome binding sites and enzyme variants. A simultaneous fermentation and extraction of astaxanthin by isopropyl myristate was performed which was supplemented to the culture medium. The bioreactor worked in a fed-batch process and isopropyl $\beta$-D-1-thiogalactopyranoside was used to induce carotenogenesis. Using all of the above methods, the production of the enantiomer of 3S, 3'S - astaxanthin of $184 \mathrm{mg} \mathrm{L}^{-1}$ day-1 or $320 \mathrm{mg} \mathrm{L}^{-1}$ (Zhang et al., 2018) was achieved.

Tramontin et al. (2019) described a genetically modified yeast Yarrowia lipolytica producing astaxanthin in a yield of $285 \mathrm{mg} \mathrm{L}^{-1}$ after 7 days of cultivation on a complex glucose medium. Cultivation was performed in a $1 \mathrm{~L}$ bioreactor, the initial culture volume was $0.4 \mathrm{~L}$, the fed-batch process of $50 \%$ glucose solution was started 6 hours after inoculation. The glucose concentration was kept below $5 \mathrm{~g} \mathrm{~L}^{-1}$ throughout the fermentation process. First, the authors created a strain that was optimized for the production of $\beta$-carotene, an important biosynthetic precursor of astaxanthin (see Figure 2 for astaxanthin biosynthesis). Furthermore, it included key genes encoding $\beta$-ketolase and $\beta$-hydroxylase from Paracoccus sp. and/ or Pantoea ananatis and the alga H. pluvialis.

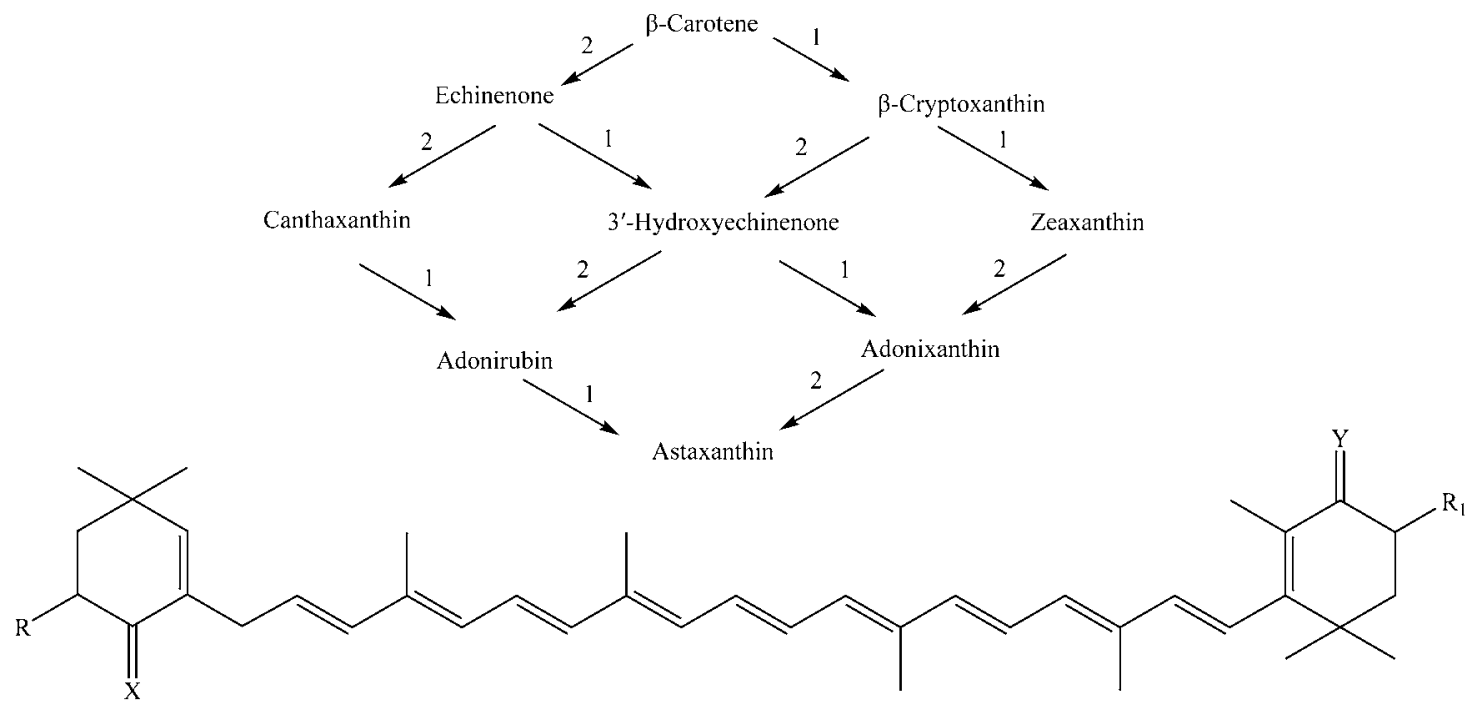

$1 \beta$-carotene ketolase

$\beta$-Carotene $X=\mathrm{H}_{2}, Y=\mathrm{H}_{2}, R=\mathrm{H}_{2}, \mathrm{R}_{1}=\mathrm{H}_{2}$
Echinenone $X=\mathrm{H}_{2}, Y=\mathrm{H}_{2}, R=\mathrm{OH}, \mathrm{R}_{1}=\mathrm{H}_{2}$

$2 \beta$-carotene hydroxylase $\beta$-Cryptoxanthin $\mathrm{X}=\mathrm{O}, \mathrm{Y}=\mathrm{H}_{2}, \mathrm{R}=\mathrm{H}_{2}, \mathrm{R}_{1}=\mathrm{H}_{2}$ Canthaxanthin $\mathrm{X}=\mathrm{O}, \mathrm{Y}=\mathrm{H}_{2}, \mathrm{R}=\mathrm{H}_{2}, \mathrm{R}_{1}=\mathrm{OH}$ 3'-Hydroxyechinenone $\mathrm{X}=\mathrm{H}_{2}, \mathrm{Y}=\mathrm{H}_{2}, \mathrm{R}=\mathrm{OH}, \mathrm{R}=\mathrm{OH}$ Zeaxanthin $\mathrm{X}=\mathrm{O}, \mathrm{Y}=\mathrm{O}, \mathrm{R}=\mathrm{H}_{2}, \mathrm{R}_{1}=\mathrm{H}_{2}$ Adonirubin $\mathrm{X}=\mathrm{H}_{2}, \mathrm{Y}=\mathrm{O}, \mathrm{R}=\mathrm{OH}, \mathrm{R}_{1}=\mathrm{OH}$ Adonixanthin $\mathrm{X}=\mathrm{O}, \mathrm{Y}=\mathrm{O}, \mathrm{R}=\mathrm{OH}, \mathrm{R}_{1}=\mathrm{H}_{2}$ Astaxanthin $\mathrm{X}=\mathrm{O}, \mathrm{Y}=\mathrm{O}, \mathrm{R}=\mathrm{OH}, \mathrm{R}_{1}=\mathrm{OH}$

Figure 2 Astaxanthin biosynthetic pathway in X. dendrorhous. 
A pilot plant process for the production of astaxanthin by fermentation of $X$. dendrorhous has been developed. In an $800 \mathrm{~L}$ fermenter, up to $350 \mathrm{mg}$ of $\mathrm{L}^{-1}\left(4.1 \mathrm{mg} \mathrm{g}^{-1}\right)$ astaxanthin was achieved by optimizing the culture conditions, i.e. by UV-light illumination and by optimizing the glucose flow. The resulting mixture contained $84 \%$ astaxanthin, $4 \% \beta$-carotene, $4 \%$ canthaxanthin, $5 \% 3$-hydroxy-3', $4^{\prime}$-didehydro- $\beta, \varphi$-carotene-4-one, $2 \%$ phoenicoxanthin and $1 \%$ zeaxanthin as minor components. This pilot plant technology can easily be extended to an industrial application for the production of this xanthophyll, which is now in high demand (de la Fuente et al., 2010).

Industrial fermentation of $X$. dendrorhous to determine astaxanthin production was performed in a 10,000 L fermenter (Zheng et al., 2006). It has been reported that the astaxanthin content of the cells reached a maximum of $52.32 \mathrm{mg} \mathrm{L}^{-1}$ and $2.57 \mathrm{mg}$ per g dry weight after 156 hours of culture.

\section{Conclusion}

Yeast having the red astaxanthin pigment has caused considerable biotechnological interest. It serves as a natural source of astaxanthin in the diet for coloring salmon, lobsters and egg yolk in poultry. The excellent antioxidant properties of astaxanthin are also commercially attractive to the pharmaceutical, cosmetic and food industries. Although all-trans astaxanthin can be produced synthetically, interest in Phaffia/Xanthophyllomyces as one of the most promising natural sources of this pigment will certainly continue in the future.

\section{Acknowledgement}

This research was supported by the Ministry of Education, Youth and Sports of the Czech Republic (L01509).

\section{References}

Alcaíno, J., Barahona, S., Carmona, M., Lozano, C., Marcoleta, A., Niklitschek, M., Sepúlveda, D., Baeza, M., Cifuentes, V. (2008). Cloning of the cytochrome p450 reductase (crtR) gene and its involvement in the astaxanthin biosynthesis of Xanthophyllomyces dendrorhous. BMC Microbiology, 8, 169. https://doi. org/10.1186/1471-2180-8-169

Alcaíno, J., Fuentealba, M., Cabrera, R., Baeza, M., Cifuentes, V. (2012). Modeling the interfacial interactions between CrtS and CrtR from Xanthophyllomyces dendrorhous, a P450 system involved in astaxanthin production. Journal of Agricultural and Food Chemistry, 60, 8640-8647. https://doi.org/10.1021/jf302287f80

Ambati, R.R., Phang, S.M., Ravi, S., Aswathanarayana, R.G. (2014). Astaxanthin: Sources, extraction, stability, biological activities and its com- mercial applications-A review. Marine Drugs, 12, 128-152. https:// doi.org/10.3390/md12010128

An, G.H., Schuman, D.B., Johnson, E.A. (1989). Isolation of Phaffia rhodozyma mutants with increased astaxanthin content. Applied and Environmental Microbiology, 55(1), 116-124.

An, G.H., Chang, K.W., Johnson, E.A. (1996). Effect of oxygen radical and aeration on carotenogenesis and growth of Phaffia rhodozyma (Xanthophyllomyces dendrorhous). Journal of Microbiology Biotechnology, 6, 103-109.

Britton, G., Liaaen-Jensen, S., Pfander, H. (1996). Carotenoids, Volume 2: Synthesis. Birkhäuser Basel. https://doi.org/10.1007/978-3-0348-9323-7

Chun, S.B., Chin, J.E., Bai, S., An, G.H. (1992). Strain improvement of Phaffia rhodozyma by protoplast fusion. FEMS Microbiology Letters, 93, 221-226. https://doi-org.ezproxy.techlib. cz/10.1111/j.1574-6968.1992.tb05101.x

de la Fuente, J.L., Rodríguez-Sáiz, M., Schleissner, C., Díez, B., Peiro, E., Barredo, J.L. (2010). High-titer production of astaxanthin by the semi-industrial fermentation of Xanthophyllomyces dendrorhous. Journal of Biotechnology, 148, 144-146. https://doi.org/10.1016/j. jbiotec.2010.05.004

Elwan, H., Elnesr, S., Abdallah, Y., Hamdy, A., El-Bogdady, A. (2019). Red yeast (Phaffia rhodozyma) as a source of astaxanthin and its impacts on productive performance and physiological responses of poultry. World's Poultry Science Journal, 75(2), 273-284. https://doi. org/10.1017/S0043933919000187

Fang, T.J., Chiou, T.Y. (1996). Batch cultivation and astaxanthin production by a mutant of the red yeast, Phaffia rhodozyma NCHUFS501. Journal of Industrial Microbiology \& Biotechnology, 16, 175-81. https://doiorg.ezproxy.techlib.cz/10.1007/BF01570001

Gassel, S., Schewe, H., Schmidt, I., Schrader, J., Sandmann, G. (2013). Multiple improvement of astaxanthin biosynthesis in Xanthophyllomyces dendrorhous by a combination of conventional mutagenesis and metabolic pathway engineering. Biotechnology Letters, 35, 565-569. https://doi.org/10.1007/s10529-012-1103-4

Gassel, S, Breitenbach, J., Sandmann, G. (2014). Genetic engineering of the complete carotenoid pathway towards enhanced astaxanthin formation in Xanthophyllomyces dendrorhous starting from a high-yield mutant. Applied and Environmental Microbiology, 98(1), 345-50. https://doi.org/10.1007/s00253-013-5358-z

Gu, W.L., An, G.H., Johnson, E.A. (1997). Ethanol increases carotenoid production in Phaffia rhodozyma. Journal of Industrial Microbiology \& Biotechnology, 19, 114-117. https://doi-org.ezproxy.techlib. cz/10.1038/sj.jim.2900425

Gutiérrez, M.S., Rojas, M.C., Sepúlveda, D., Baeza, M., Cifuentes, V., Alcaíno, J. (2015). Molecular characterization and functional analysis of cytochrome b5 reductase (CBR) encoding genes from the carotenogenic yeast Xanthophyllomyces dendrorhous. PLoS One, 10:e0140424. https://doi.org/10.1371/journal.pone.0140424

Jin, J., Wang, Y., Yao, M., Gu, X., Li, B., Liu, H., Ding, M., Xiao,W., Yuan, Y. (2018). Astaxanthin overproduction in yeast by strain engineering and new gene target uncovering. Biotechnology for Biofuels, 11, 230. https://doi.org/10.1186/s13068-018-1227-4

Kildegaard, K.R., Adiego-Pérez, B., Doménech Belda, D., Khangura, J.K., Holkenbrink, C., Borodina, I. (2017). Engineering of Yarrowia lipolytica for production of astaxanthin. Synthetic and Systems Biotechnology, 2, 287-294. https://doi.org/10.1016/ j.synbio.2017.10.002

Kim, S.J., Kim, G.J., Park, D.H., Ryu, Y.W. (2003). High-level production of astaxanthin by fed-batch culture of mutant strain Phaffia rhodozyma AJ-6-1. Journal of Microbiology and Biotechnology, 13, 175-181.

Liang, C., Zhao, F., Wei, W., Wen, Z., Qin, S. (2006). Carotenoid biosynthesis in Cyanobacteria: structural and evolutionary scenarios based on comparative genomics. International Journal of Biological Sciences, 2(4), 197-207. https://doi.org/197-207.10.7150/ijbs.2.197 
Lockwood, S.F., Tang, P.Ch., Nadolski, G., Jackson, H.L., Fang, Z., Du, Y., Yang, M., Geiss, W., Williams, R., Burdick D. (2007). Methods for the synthesis of astaxanthin. US7247752B2.

Lorenz, R.T., Cysewski, G.R. (2000). Commercial potential for Haematococcus microalgae as a natural source of astaxanthin. Trends in biotechnology, 18(4), 160-167. https://doi.org/10.1016/S01677799(00)01433-5

Mata-Gomez, L.C., Montanez, J.C., Mendez-Zavala, A., Aguilar, C.N. (2014). Biotechnological production of carotenoids by yeasts: An overview. Microbial Cell Factories, 13, 12. https://doi.org/10.1186/14752859-13-12

Meyer, P.S., du Preez, J.C. (1994). Astaxanthin production by a Phaffia rhodozyma mutant on grape juice. World Journal of Microbiology and Biotechnology, 10, 178-183. https://doi-org.ezproxy.techlib. cz/10.1007/BF00360882

Ojima, K., Breitenbach, J., Visser, H., Setoguchi, Y., Tabata, K., Hoshino, T., van den Berg, J., Sandmann, G. (2006). Cloning of the astaxanthin synthase gene from Xanthophyllomyces dendrorhous (Phaffia rhodozyma) and its assignment as a beta-carotene 3-hydroxylase/4ketolase. Molecular Genetics and Genomics, 275(2), 148-158. https://doi.org/10.1007/s00438-005-0072-X

Park, S.Y., Binkley, R.M., Kim,W.J., Lee, M.H., Lee, S.Y. (2018). Metabolic engineering of Escherichia coli for high-level astaxanthin production with high productivity. Metabolic Engineering, 49, 105-115. https:// doi.org/10.1016/j.ymben.2018.08.002

Reynders, M.B., Rawlings, D.E., Harrison, S.T.L. (1997). Demonstration of the Crabtree effect in Phaffia rhodozyma during continuous and fedbatch cultivation. Biotechnology Letters, 19(6), 549-552. https:// doi-org.ezproxy.techlib.cz/10.1023/A:1018341421122

Rezanka T., Nedbalova L., Kolouchova I., Sigler K. (2013). LC-MS/APCI identification of glucoside esters and diesters of astaxanthin from the snow alga Chlamydomonas nivalis including their optical stereoisomers. Phytochemistry, 88, 34-42. https://doi.org/10.1016/j. phytochem.2013.01.003

Sandmann, G. (2002). The mycota X industrial applications. In: Karl Esser PAL, Bennett JW, Heinz D, editors. The Mycota X industrial applications. Osiewacz: Springer Verlag Berlin.

Schmidt I, Schewe H, Gassel S, Jin C, Buckingham J, Hümbelin M, Sandmann G, Schrader J. (2011). Biotechnological production of astaxanthin with Phaffia rhodozyma/Xanthophyllomyces dendrorhous. Applied Microbiology and Biotechnology, 89, 555-571. https://doi. org/10.1007/s00253-010-2976-6

Schroeder, W.A., Johnson, E.A. (1993). Antioxidant role of carotenoids in Phaffia rhodozyma. Journal of General Microbiology, 139, 907-912. https://doi.org/10.1099/00221287-139-5-907

Schroeder, W.A., Johnson, E.A. (1995). Singlet oxygen and peroxyl radicals regulate carotenoid biosynthesis in Phaffia rhodozyma. The Journal of Biological Chemistry, 270, 18374-18379. https://doi. org/10.1074/jbc.270.31.18374
Steinbrenner, J., Sandmann, G. (2006). Transformation of the green alga Haematococcus pluvialis with a phytoene desaturase for accelerated astaxanthin biosynthesis. Applied and Environmental Microbiology, 72, 7477-7484. https://doi.org/10.1128/AEM.01461-06

Tramontin, L. R. R., Kildegaard, K. R., Sudarsan, S., Borodina I. (2019). Enhancement of astaxanthin biosynthesis in oleaginous yeast Yarrowia lipolytica via microalgal pathway. Microorganisms, 7(10), 472. https://doi.org/10.3390/microorganisms7100472

Vachali, P., Bhosale, P., Bernstein, P.S. (2012). Microbial Carotenoids. In: Barredo JL. (eds) Microbial Carotenoids From Fungi. Methods in Molecular Biology (Methods and Protocols), vol 898. Humana Press, Totowa, NJ.

Werner, N., Gómez, M., Baeza, M., Cifuentes, V., Alcaíno, J. (2016). Functional characterization of thiolase-encoding genes from Xanthophyllomyces dendrorhous and their effects on carotenoid synthesis. BMC Microbiology, 16, 278. https://doi.org/ 10.1186/s12866-016-0893-2

William, A., Schroeder, E.A.J. (1993). Antioxidant role of carotenoids in Phaffia rhodozyma. Microbiology, 139(5), 907-12.

William, A., Schroeder, E.A.J. (1995). Carotenoids protect Phaffia rhodozy$m a$ against singlet oxygen damage. Journal of Industrial Microbiology \& Biotechnology, 14(6), 502-7. https://doi-org.ezproxy.techlib. cz/10.1007/BF01573965

Zhang, C., Seow, V.Y., Chen, X., Too, H.P. (2018). Multidimensional heuristic process for high-yield production of astaxanthin and fragrance molecules in Escherichia coli. Nature Communications, 9, 1858. https:// doi.org/10.1038/s41467-018-04211-x

Zheng,Y.G., Hu,Z.C., Wang, Z., Shen, Y.C. (2006). Large-scale production of astaxanthin by Xanthophyllomyces dendrorhous. Food and Bioproducts Processing, 84(2), 164-166. https://doi.org/10.1205/fbp.05030

Zhou, P., Ye, L., Xie, W., Lv, X., Yu, H. (2015). Highly eficient biosynthesis of astaxanthin in Saccharomyces cerevisiae by integration and tuning of algal crtZ and bkt. Applied Microbiology and Biotechnology, 99(20), 8419-8428. https://doi.org/10.1007/s00253-015-6791-y

Zhou, P., Xie, W., Li, A.,Wang, F., Yao, Z., Bian, Q., Zhu, Y., Yu, H., Ye, L. (2017). Alleviation of metabolic bottleneck by combinatorial engineering enhanced astaxanthin synthesis in Saccharomyces cerevisiae. Enzyme and Microbial Technology, 100, 28-36. https://doi.org/10.1016/j. enzmictec.2017.02.006 